\title{
Simulating Acoustic Emission: the Noise of Collapsing Domains
}

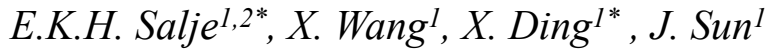 \\ 1. State Key Laboratory for Mechanical Behavior of Materials, Xi'an Jiaotong University, \\ Xi'an 710049, China \\ 2. Department of Earth Sciences, University of Cambridge, Cambridge CB2 3EQ, UK \\ *Corresponding authors: ekhard@esc.cam.ac.uk; dingxd@mail.xjtu.edu.cn
}

\begin{abstract}
Microstructural changes during mechanical shear of a ferroelastic or martensitic material and their signature in acoustic emission (AE) spectroscopy during strain induced yield and detwinning are investigated by computer simulation. Complex domain patterns are generated during the main yield event, which leads to large displacements of surface atoms and strong emission of acoustic waves. Loading beyond the yield point leads, eventually, to a simplification of the domain patterns by local movements of needles domains, the nucleation and movement of kinks in domain walls, and the collapse of domains spanning the entire sample (from surface to surface). These microstructural changes lead to much weaker acoustic emissions than those near the yield point. Nucleation/collapse during a yield event involves energies of some $3.7 \mathrm{meV} /$ atom, the collapse of spanning domains releases $0.56 \mathrm{meV} /$ atom, a kink crashing into the surface changes the energy by $0.017 \mathrm{meV} / \mathrm{atom}$, and the collapsing vertical needle by $0.016 \mathrm{meV} /$ atom. All these energy bursts can, in principle, be seen by AE. The large energy spread means that AE spectroscopy measures a mixture of events whereby weak and strong signals may signify smaller and bigger events of the same kind or different microstructural changes with intrinsically different signal strengths. In order to disentangle the various contributions, other observables are needed, such as the time dependent strain matrix of the deformed sample.
\end{abstract}




\section{INTRODUCTION}

Acoustic emission (AE) spectroscopy is one of the most powerful experimental techniques for the examination of jerk movements under changing fields, temperature or pressure ${ }^{1-22}$. Jerks are short bursts; they are distinct from continuous movements such as those originating from phonons and continuous propagation of domain boundaries. Jerks are often associated with crackling noise and the formation of avalanches ${ }^{7,23}$. A simple connection between the experimental signal and the underlying physical process is not straightforward, however ${ }^{23}$. Mixtures of continuous and jerky movements of domains were observed in martensites ${ }^{14,15}$ which shows that not all microstructural changes lead to jerks. No jerks occur if smooth domain movements compensate the applied strain. Such smooth movements are typical for the large group of adaptive structures ${ }^{12}$ where the interfacial energy is small and where domain movements are virtually unpinned.

Jerks can also be observed when no avalanches exist. Examples are repeated pinning / depinning processes of a ferroelastic needle domain (serration), which generate several large jerks and cannot be distinguished from the more complex formation of avalanches where many depinning movements interact ${ }^{24}$. In different systems, jerks have been detected with a variety of other experimental techniques: induction by magnetic Barkhausen jumps ${ }^{25,26}$, magnetization measurements ${ }^{27}$, calorimetry ${ }^{15,28}$, resistivity ${ }^{29,30}$ and capacitance measurements ${ }^{31}$, and optical observations $^{24,32}$. In comparison with these techniques, $\mathrm{AE}$ appears to be the most popular method for the observation of intense jerks with over 1500 publications per year in physics and material science journals. Despite this colossal effort it remains unclear which atomic processes are observed in AE experiments.

The obvious application of AE relates to sudden volume changes $18,33,34$. The collapse of cavities in porous materials under stress leads to very large AE signals ${ }^{7,14,16-18}$. Weaker AE signals occur 
for microstructural changes in martensites and ferroelastics. AE signals were found in $\mathrm{Cu}_{67.64} \mathrm{Zn}_{16.71} \mathrm{Al}_{15.65}$ where the volume strain is -0.003 in Ref.15, which is large compared with most ferroelastic materials where the volume strain is typical below $10^{-4}$. The spontaneous strain of the martensitic transformation B2- $\mathrm{R}$ in Ni-Ti-Fe is 0.016 in Ref.35, which is similar to oxide and fluorite materials such as $\mathrm{KMnF}_{3}$. These materials show de-pinning jerks ${ }^{36,37}$ but not in $\mathrm{KMnF}_{3}$ so that it seems that strain jerk of ca. 0.001 represents the lower limit for the sensitivity of current $\mathrm{AE}$ experiments.

It is purpose of this paper to show how various ferroelastic materials show microstructural changes and how these changes lead to jerks. The jerks are measured at the surface of the sample similar to a 'real' experiment: the sudden movements of surface atoms transmit the incoming acoustic wave to the transducer, which is attached to the surface. We simulate a sheared ferroelastic structure with a shear angle of $4^{\circ}$ and a spontaneous strain $e_{s}=(x-y) / x=0.072$ (7.2\%). This value is within the experimental range of martensitic and ferroelastic materials with large deformations, and have been observed in AE experiments. We will show that the change of the micro-structure under (de-) compression (at constant temperature) can lead to large AE signals from three different origins: the nucleation of twin boundaries, the propagation of kinks inside twin boundaries and the annihilation of needle domains. In addition, we show how a simple yield point stress release leads to very strong and complex AE signals. We focus in the paper on the displacement of surface atoms on two opposite sides of the sample (two lines in a two-dimensional model). Additional AE signals will emerge from other faces of the sample if the sample is much thicker than in our simulations. We ignore this effect although it will add to the I simulated displacements and increases the AE signals.

The paper is organized as follows: first we describe the model, then we describe the three scenarios of basic changes of the microstructure and discuss their effect of jerks at the sample surface as it would be seen in AE. Finally we show the results for the yield point collapse.

\section{THE MODEL}


We simulate the microstructural changes by a well-established model for ferroelastic transitions based on spring mediated interactions ${ }^{38}$. The model used a generic two-body potential to represent the interactions of atoms in two-dimensional (2D) system. The potential energy $U(r)$ contains three parts, as the first-nearest atomic interactions of $20(r-1)^{2}$, the second-nearest interactions $-10(r-\sqrt{2})^{2}+2000(r-\sqrt{2})^{4}$ and the third-nearest interactions $-(r-2)^{4}$, where $r$ is atomic distance. This potential is developed based on Landau theory by choosing the shear angle as "order parameter". The details of properties obtained by this potential are described in our previous work ${ }^{38-44}$. Extensions of this model to three dimensions did not change the principle results of the simulations ${ }^{45}$ so that we make use of the higher efficiency of two-dimensional simulations.

The equilibrated unit cell has the shape of parallelogram with the shear angle of 4 degrees. We set the equilibrium lattice constant $a=1 \AA$ and atomic mass to $M=100 \mathrm{amu}$. The initial configuration contains two horizontal twin boundaries (HTBs). The surface ratio of intermediate

I layer to the whole sample is fixed to be 0.5. The size of the present simulations are all based on $a_{4} 400 a \times 402 a$ box except a $200 a \times 202 a b o x$ is used to capture the collapse of one single vertical needle domain under de-twinning conditions. The external load was applied to the top and bottom 3 layers, which were fixed rigidly as the loading grip. The system was first relaxed using a conjugate gradient refinement procedure to find the optimal position for each lattice point under the initial conditions of the sample shape. Molecular Dynamics (MD) was then performed to anneal each configuration at a given temperature for $3 \times 10^{6}$ time steps. The only relaxations, which occurred during this procedure, were surface relaxations. After the relaxation and strainfree MD, external strain was applied via a global shear of the two boundary layers. We use a constant strain rate of $10^{-5} / \mathrm{ps}$ and display our results as function of time to directly connect with the dynamics of acoustic emission. The temperature of the sample was held at $\mathrm{T}=0.6 \mathrm{~K}$ by the Nosé-Hoover thermostat ${ }^{46,47}$. All the calculations are performed using the Large-scale Atomic/ Molecular Massively Parallel Simulator, LAMMPS, $\operatorname{code}^{48}$.

\section{RESULTS}


The time evolution of the domain formation and the de-twinning sequence is shown on a long time scale in Fig.1 and Fig.2 presents the corresponding energy evolution. The initial crystal (Fig.1(a)) is heavily twinned during a yield event between time $t_{1}$ when the first twin nucleates and time $t_{2}$ when the external strain is compensated by the shape change of the sample. The crystal decays into a multitude of twins (Fig.1(b)). The twinned area then decreases under further shear ( Fig.1(c)-(e)), and a single crystal is recovered in Fig.1(f). The acoustic emission signal is largest during the yield event, where most experimental results were obtained. During the yield event a complex mixture of domain movements will occur with needle domains, kinks and junctions all forming almost simultaneously. During de-twinning, the same movements occur but they are spread out over a long time period. The $\mathrm{AE}$ of each event is much less than the total $\mathrm{AE}$ during yield but, if the de-twinning AE could be accumulated in full, would lead to the same energy change. We will now analyze the elementary movements of the twinning and de-twinning

I processes and compare the energies with those of the yield event.

\section{A. Collapse of spanning needle domains in the horizontal direction}

The formation and destruction of needle microstructures is motivated by the experimental observations in Ref.21 where the close relationship between needle formation and propagation, and $\mathrm{AE}$ was demonstrated. The de-twinning of a horizontal needle domain at the high strain end I of the plastic regime (region C in Fig.2) is shown in Fig.3. The mechanism of the disappearance of the spanning domain consists of 3 parts. First, some vertical needles nucleate inside the horizontal domain. These vertical needles then move laterally until gaps appear in the horizontal needle domain. Finally the segments of the horizontal needle(s) shrink and disappear so that the single domain state is established (Fig. 3).

The collapse of the potential energy during the final de-twinning is large $(0.56 \mathrm{meV} /$ atom in Fig. 4). Only a small part of this energy leads to increased thermal vibrations and can be seen as an increase of the kinetic energy. The main part of the potential energy leads to atomic displacements, which propagate to the surface and are observed as AE signal. The displacements of two surface atoms during the final collapse of the horizontal domain are shown in Fig.5. The spanning domain is 5 atomic layers thick ( $5 \AA$ ). One reference atom is situated at the uppermost 
I layer of the domain $\left(\mathrm{C}_{1}\right.$ in Fig.5(a)), the other atom belongs to the lowest layer( $\mathrm{C}_{2}$ in Fig.5(a)). The displacements of these two atoms in the vertical y-direction (along the surface layer) are I essentially the same. The displacements in the x-direction (perpendicular to the surface layer) is I antiparallel with the displacement of the atom further away from the rigid loading grip $\left(\mathrm{C}_{1}\right)$ is

I $-0.448 \AA$ and the displacement of the atom near the rigid loading grip $\left(C_{2}\right)$ is $0.256 \AA$. The shear strain across the domain is them $0.7 \AA / 5 \AA=0.14$ which is the same as the macroscopic shear of the relaxed sample.

\section{B. Collision of kinks with the surface}

A very common dynamics for the advance of a domain wall is the formation of a kink (or latch) inside the domain wall. When the kink has covered the twin plane it will have advanced (or retracted) the twin wall by one atomic layer. The kink moves to the surface. In a two-dimensional pattern the movement of the twin wall by one layer is completed when the kink hits the surface. Further movements require the nucleation of another kink. In three dimensions the movement is often by spirals, not dissimilar to growth spirals during crystal growth. These kinks were first anticipated in polytypic $\mathrm{PbI}_{2}$ (Ref.49) and commonly observed in computer simulations of I moving domain walls ${ }^{38-45}$. A moving kink (corresponding to region B in Fig.2) is shown in Fig.6. Fig.6(a) shows the kink in the bulk of the materials, in Fig.6(b) the kink has moved to the surface while in Fig.6(c) the collision process has finished. The colors of the atoms show the local shear angle.

The moving kink emits phonons while the impact at the surface leads to a very large distortion of the crystal structure near the impact point. The shift of the atomic positions during the impact of Fig.6(a) and (c) is shown in Fig.6(d). Two position patterns are shown which evolve from one to the other in $0.8 \mathrm{ps}$. The atomic shifts are very similar in the bulk and at the twin boundary, although some small differences exist due to surface relaxations. The displacements of the two atoms B1 and B2 in Fig. 6(b) and 6(d) are shown in Fig.7. The relative displacement between the 
atoms in $\mathrm{x}$ direction is $0.14 \AA$, the surface strain is hence $14 \%$. The velocity of the movement is ca. $30 \mathrm{~m} / \mathrm{s}$, which is within the range of acoustic surface waves. The energy changes during the I crash are shown in Fig.8.

\section{Irreversible collapse of a vertical needle domain under de-twinning conditions}

De-twinning is simulated during the decrease of the applied shear strain. The initial condition is the sandwich model with one vertical needle domain between the two horizontal needles. The temperature of the sample is $0.6 \mathrm{~K}$. Strain release changes the length of the needle domain until the irreversibility point is reached where the length of the needle no longer follows the external shear strain ${ }^{41}$ but collapses irreversibly. Previous work has shown that the irreversibility point is passed when the length of the needle domain is shorter than half the distance between the two limiting domain boundaries ${ }^{41}$. At this point the needle shrinks rapidly, even without additional driving force by the external shear strain. Thermal fluctuations alone are sufficient to lead to a rapid collapse of the needle, which is annihilated only a few picoseconds after the irreversibility point is passed. In the simulation we drive the de-twinning process by strain release to the irreversibility point and then keep the external strain constant. We then anneal the sample at constant temperature. The collapse occurs during this annealing process. The change of the potential energy is shown as function of annealing time in Fig.9.

The sudden collapse of the potential energy by $0.016 \mathrm{meV} /$ atom is clearly visible. The time when the isothermal anneal leads to the first rapid shrinking of the needle $\mathrm{P}_{2}$ and the time when the needle domain has completely collapsed $\mathrm{P}_{4}$ are indicated in this figure. Both points are situated

I well before the collapse, the incubation time is $5.8 \mathrm{ps}$ \(the time to dissipate the potential energy I into the sample). The typical microstructures for the same time interval are shown in Fig.9. The collapse of the needle leads to the emission of shock waves, which propagate through the sample I (upper figures $\mathrm{P}_{4}-\mathrm{P}_{6}$ in Fig.9). Simultaneously we see a sharp increase of lattice vibrations when the shock wave reaches the sample surface. The vibration of an atom in the middle of the surface I is shown in Fig.10(a). The equilibrium phonon vibrations at the beginning of the simulation 
suddenly increase twice when the potential energy of the sample collapses and the energy is transferred from potential energy to vibrational energy.

We now analyze the vibrational spectra in more detail. The question is whether the frequencies of the lattice vibrations change with the collapse or whether only the vibrational amplitudes increase. The frequency spectrum of the fluctuations of the surface atom is related to the dynamic susceptibility via the fluctuation-dissipation theorem(FDT $)^{51}$. The spectrum $\alpha "(\omega)$ is determined by the Fourier transform of the displacement autocorrelation function $\mathrm{A}(\omega)=\mathrm{FT}<\mathrm{x}$ (t) $\mathrm{x}(0)>$ by $\mathrm{A}(\omega) \sim \alpha " \hbar \omega$ coth $(\hbar \omega / 2 \mathrm{kT})$. Two spectra $\mathrm{S}(\omega)=\omega \mathrm{A}(\omega)$ before and after the I collapse are shown in Fig.10(b). Both spectra have essentially the same frequencies. The effect of the ringing of the sample after the collapse is hence to increase the vibrational intensities but maintains the same spectral distribution. In particular the modes near $0.55 \mathrm{THz}$ and $0.7 \mathrm{THz}$ are heavily excited. This result predicts that the collapse of needle domains should be visible in Raman spectra where each collapse leads to a short increase (a spike) of Raman intensity.

Similarly, we find that the displacement amplitudes of the surface atoms increase together with the increase of $S(\omega)$. This effect is often described as phonon pressure. In our case the phonon pressure is a short burst which is equivalent to a sudden increase of temperature and the equivalent thermal expansion of the sample. The increase of temperature during the burst is roughly twice. We can estimate the thermal expansion effect in a typical metal with a thermal expansion coefficient of $10^{-5} \mathrm{~T}^{-1}$ as a change between room temperature $(300 \mathrm{~K})$ and the burst temperature $(600 \mathrm{~K})$ as being equivalent to an expansion of $3 \%$ of the sample. This sudden expansion jerk is large enough to be seen in AE experiments.

I The decay of the potential energy before the critical collapse is shown in more detail in Fig.11. The short interval between the initial shrinking of the needle domain and the final collapse of the potential energy takes some $5.8 \mathrm{ps}$. During this time the equilibration of the thermal energy takes place. The energy decay shows a large precursor effect before the needle collapse. The potential I energy in this regime decays with increasing annealing time from the background regime where 
only the thermally equilibrated vibrations exist to the ringing regime where the released potential I energy of the needle domains is converted to vibrational energy. The time dependence in Fig. 11. follows in good approximation $\Delta \mathrm{E} \sim \log { }^{1 / 2}\left(\mathrm{t}-\mathrm{t}_{\mathrm{o}}\right)$, where $\Delta \mathrm{E}$ is the energy difference between the potential energy and the potential energy when the needle has collapsed $t_{0}\left(t_{0}=P_{4}\right.$ in the present case). The time is measured as time difference between the time $t$ and the time $t_{0}$ when the needle has fully collapsed. Note that this dependence does not represent the softening after the collapse (as expressed usually as power laws and a softening exponent) but represents a precursor energy release. We are not aware of any analytical predictions for this effect. The role of precursor softening was previously observed in compressed porous materials ${ }^{17,18}$ but not in de-pinning processes or in martensites. The time interval of both the precursor regime and the actual collapse (the emission of a shock wave) is extremely short and cannot be resolved in AE experiments.

The length of the time interval after the collapse in which the enhanced vibrations can be observed does not depend on the time scale of the collapse but is entirely determined by the energy dissipation of the ringing event. This clarifies a key question in avalanche research: the profile of the jerks as avalanche signal has nothing to do with the physical event of the individual needle collapse but depends on the friction of the excited waves (the ringing) and the distribution of such events during larger avalanches. Individual spikes in AE are somewhat 'artifacts' of the transmitting medium and contains no information about the individual physical process which operates on a much shorter time scale than can be observed in AE.

\section{Acoustic emission during the yield event}

The main acoustic emission will happen when the strain passes the yield point. The yield point is characterized by the nucleation of a complex domain pattern, which consists of needle domains, spanning domain boundaries and kinks inside domain boundaries. The yield event is often visible in $\mathrm{AE}$ experiments and constitutes the 'big bang' in ferroelastic and martensitic materials. We have chosen the temperature and boundary conditions such that the resulting yield pattern 
remains relatively simple ${ }^{43}$ and the lattice distortions are easy to follow in the computer simulation. The yield pattern is shown in Fig.1 (b) where needle and kink domains are clearly visible. The main difference between the de-twinning scenarios (described above) and the yield I event (region A in Fig.2) is the different energy partition between static wall energies and vibrational energies. The phonon energy in the twinning case decays very rapidly because the excess potential energy is transferred to the twin boundary energy while no such twin boundaries exist in the de-twinning case so that the kinetic energy leads to a long time 'ringing' of the sample. The phonon pressure is hence smaller during nucleation while the static deformation is much stronger. The atomic displacements after the yield event are shown in Fig. 12(a) and the detailed displacements in $\mathrm{x}$ and $\mathrm{y}$ direction of the surface atoms are shown in Figs.12(b) and (c).

I These displacements are around $1.8 \AA$ in $\mathrm{x}$ direction over the entire surface and are expected to lead to large AE signals, surpassing all displacements by individual needle domains and kinks. This signal combines several individual movements and can only be used as a guide to the nucleation of a complex pattern. It is explicitly size dependent and will, for large enough sizes, scale as the volume of the sample. The time evolution depends on the complexity of the avalanches. In our relatively small computer simulation we find a collapse time of some picoseconds, this time will greatly lengthen if several avalanches of this type conspire to yield much larger avalanches.

The energy release of potential energy of the entire sample is shown in Fig. 13(a) and (b). The total energy reduction is ca. $3.7 \mathrm{meV} /$ atom and occurs over an initial time interval of ca. $14 \mathrm{ps}$.

I The relaxation tail in Fig.13(a) extends than over some 17ps. The drop in potential energy is partly compensated by the interfacial energy of the twin domains and partly by the kinetic energy I in Fig. $13(\mathrm{~b})$, The kinetic energy increases at the step by $0.09 \mathrm{meV} /$ atom while the remaining $3.6 \mathrm{meV} /$ atom is stored in additional interfacial energy.

\section{Discussion}

The AE signals in martensites and ferroelastic materials under stress are composed of several events. They occur under strain increase (twinning) and strain release (de-twinning). The main 
event is an avalanche generated at the yield point. The avalanche energy is an extensive quantity for large samples. In our simulation, a very small avalanche releases some $3.7 \mathrm{meV} /$ atom, which is already a significant energy for AE. Most of this energy is consumed by lattice distortions and surface energies of the nucleating twin boundaries. Only a small part leads to increased vibrational amplitudes (ringing of the sample). Avalanches of this kind are the same as those generated during the phase transformation and have been observed experimentally ${ }^{7}$. All other

I events are part of such avalanches both during twinning and de-twinning. The energy release, for kinks, vertical needles and horizontal (spanning) domains is much smaller than the yield energy. This means that the energy of the pattern formation at the yield point corresponds to more than 10 elementary events. Visual inspection of the yield pattern seems to confirm this estimate.

Ringing during de-twinning, when energy is transferred into vibrational energy, will be dampened in real systems by internal friction. We did not consider friction in our simulations because damping times are generally longer than our run times. We always find heat spikes near the collapse point. These heat spikes have similar energies as those in AE signals, which are determined by the longitudinal displacements of the surface atoms. These displacements are up to $1.8 \AA$ for the major yield event, $0.7 \AA$ for the collapse of the horizontal needle, $0.14 \AA$ for the kink crashing into the surface, and $0.0014 \AA$ for the collapsing vertical needle. The equivalent energy releases are 3.7, 0.56, 0.017 and $0.016 \mathrm{meV} /$ atom, respectively. The data are not thermodynamically averaged so that the scatter is rather large. In addition, our sample is too small to compare their scaling with conclusion drawn from experimental data in Ref.15. I Nevertheless, we presume that the energy releases are proportional to the integrated AE signals of the various events. The proportionality between the AE intensity and the energy release of the sample was found experimentally in Ref.15. The individual jerks energies were experimentally obtained and calculated in previous computer simulations determined by the squared differentials of the strain dependent potential energy: $\mid \begin{aligned} & \mathrm{E}_{\text {Jerk }}=(\partial \mathrm{P} / \partial \mathrm{e})^{2}=(\Delta \sigma)^{2} \sim \text { 国E (the equiation is disorder in my PC. Please double-check it in } \\ & \text { your computer.) }\end{aligned}$ which represents the local energy drop by the stress release $\Delta \sigma$. 
In summary, we find that the yield event will always give good AE signals. The much weaker movement of kinks may also be visible in $\mathrm{AE}$ while the collapse of the vertical needle has an extremely weak signal and is unlikely to contribute to the AE signal. The borderline is expected to be the collapse of the spanning horizontal twin domain, which requires very high sensitivity and is presumably beyond most experimental resolution.

\section{ACKNOWLEDGMENT}

We appreciate the support of National Natural Science foundation of China (51171140, 51321003, 51320105014). EKHS is grateful for support by the Leverhulme fund (RG66640) and EPSRC (EP/K009702/1). 


\section{References:}

[1] S. V. Makarov, V. A. Plotnikov and A. I.Potekaev, Russ. Phys. J. 56, 630(2013).

[2] A. S. Argon, Philoso. Mag. 93, 3795(2013).

[3] S. A. Khan, P. Chivavibul, P. Sedlak, S. Arai and M. Enoki, Metall. Mater. Trans. A. 44 ,3623( 2013).

[4] M. E. Cox and D. C. Dunand, Metall. Mater. Trans. A. 44 ,3114( 2013).

[5] A. Vinogradov, A. Danyuk and V. A. Khonik, J. Appl. Phys.113,153503(2013).

[6] E. Dul'kin and M. Roth, J. Phys.: Condens. Matter 25,155901(2013).

[7] J. Baró, Á. Corral, X. Illa, A. Planes, E. K. H. Salje,W. Schranz, D. E. Soto-Parra and E. Vives, Phys. Rev. Lett. 110,088702(2013).

[8] M. J. Alava, P. K. V. V. Nukalaz and S. Zapperi, Adv. Phys. 55, 349(2006).

[9] M. Roth, E. Mojaev, E. Dul'kin, P. Gemeiner and B. Dkhil, Phys. Rev. Lett. 98,265701 (2007).

[10] M. Koslowski, R. LeSar and R.Thomson, Phys. Rev. Lett. 93,125502 (2004).

[11] T. Richeton, J. Weiss and F. Louchet, Acta. Mater. 53,4463( 2005).

[12] D.D. Viehland and E.K.H. Salje. Emergent phenomena in domain boundary dominated materials: adaptive structures and functional twin boundaries, Advances in Physics, in press.

[13] M. Minozzi, G. Caldarelli, L. Pietronero and S. Zapperi, Eur. Phys. J. B 36, 203(2003).

[14] E. K. H. Salje, J. Koppensteiner, M. Reinecker, W. Schranz and A.Planes, Appl. Phys. Lett. 95,231908 (2009).

[15] M. C. Gallardo, J. Manchado, F. J. Romero, J. Cerro, E. K. H. Salje, A. Planes, E. Vives, R. Romero and M. Stipcich, Phys. Rev. B 81,174102( 2010).

[16] E. K. H. Salje, D. E. Soto-Parra, A. Planes, E. Vives, M. Reinecker and W. Schranz, Philos. Mag. Lett. 91, 554 (2011).

[17] E. K. H. Salje, G. I. Lampronti, D. E. Soto-Parra, J. Baró, A. Planes and E. Vives, Am. Mineral. 98, 609(2013).

[18] P. O. Castillo-Villa, J. Baró, A. Planes, E. K. H. Salje, P. Sellappan, W. M. Kriven and E. Vives, J. Phys.: Condens. Matter 25,292202(2013).

[19] R. Niemann, J. Baró, O. Heczko, L. Schultz, S. Fähler, E. Vives, L. Mañosa and A. Planes, Phys. Rev. B 86, 214101 (2012).

[20] E. Vives, D. Soto-Parra, L. Mañosa, R. Romero and A. Planes, Phys. Rev. B 84 , 060101 (2011).

[21] E. Bonnot, E. Vives, L. Mañosa, A. Planes and R. Romero, Phys. Rev. B 78 , 094104 (2008).

[22] J. P. Sethna, K. A. Dahmen and C. R. Myers, Nature. 410 ,242(2001).

[23] E. K. H. Salje and K. A. Dahmen, Annu. Rev. Conden Ma P 5:233-54(2014).

[24] R. J. Harrison and E. K. H. Salje, Appl. Phys. Lett. 97, 021907 (2010).

[25] G. Durin and S. Zapperi, arXiv:cond-mat/0404512.

[26] H. Barkhausen, Phys. Z. 20, 401 (1919).

[27] V. Hardy, S. Majumdar, S. J. Crowe, M. R. Lees, D. McK. Paul, L. Hervé, A. Maignan, S. Hébert, C. Martin, C. Yaicle, M. Hervieu and B. Raveau, Phys. Rev. B 69, 020407(R) (2004).

[28] L. Carrillo and J. Ortín, Phys. Rev. B 56,11508(1997).

[29] W. Wu and P.W. Adams, Phys. Rev. Lett. 74, 610 (1995). 
[30] J. C. Lashley, K. Gofryk, B. Mihaila, J. L. Smith and E. K. H. Salje, J. Phys.:Condensed Matter 26, 035701 (2014).

[31] M. P. Lilly, P. T. Finley and R. B. Hallock, Phys. Rev. Lett. 71, 4186 (1993).

[32] D. H. Kim, S. B. Choe and S. C. Shin, Phys. Rev. Lett. 90, 087203 (2003).

[33] F. J. Pérez-Reche, E. Vives, L. Mañosa and A. Planes, Phys. Rev. Lett. 87, 195701 (2001).

[34] K. Gall, K. Jacobus, H. Sehitoglu and H. J. Maier, Metall. Mater. Trans. A. 29 ,765(1998).

[35] E. K. H. Salje, H. Zhang, A. Planes and X. Moya, J. Phys.: Condens. Matter 20,275216 (2008).

[36] E. K. H. Salje, and H. Zhang, J. Phys.: Condens. Matter 21 ,035901(2009).

[37] W. Schranz, P. Sondergeld, V. Kityk and E.K.H. Salje, Phys. Rev. B 80,094110 (2009).

[38] E. K. H. Salje, X. Ding, Z. Zhao, T. Lookman and A. Saxena, Phys. Rev. B 83,104109(2011).

[39] X. Ding, Z. Zhao, T. Lookman, A. Saxena and E. K. H. Salje, Adv. Mater. 24, 5385 (2012).

[40] E. K. H. Salje, X. Ding, Z. Zhao and T. Lookman, Appl. Phys. Lett. 100, 222905 (2012).

[41] E. K.H. Salje, Z. Zhao, X. Ding and J. Sun, Am. Mineral. 98,1449(2013).

[42] Z. Zhao, X. Ding, T. Lookman, J. Sun and E. K. H. Salje, Adv. Mater. 25,3244(2013).

[43] E. K. H. Salje,X. Ding and Z. Zhao, Appl. Phys. Lett. 102 ,152909(2013).

[44] X. Ding, T. Lookman, Z. Zhao, A. Saxena, J. Sun and E. K. H. Salje, Phys. Rev. B 87,094109 (2013).

[45] Z. Zhao, X. Ding, J. Sun and E. K.H. Salje, J. Phys.: Condens. Matter 26 , 142201 ( 2014).

[46] S. Nose, J. Chem. Phys. 81, 511 (1984)

[47] W. G. Hoover, Phys. Rev. A 31, 1695 (1985)

[48] S. Plimpton, J. Comput. Phys. 117, 1 (1995)

[49] E. K. H. Salje, B. Palosz and B. Wruck, J. Phys. C: Solid State Phys. 20 ,4077(1987).

[50] J. Novak and E. K. H. Salje, J. Phys.: Condens. Matter 10,L359(1998).

[51] L. D. Landau and E. M. Lifshitz, Statistical Physics, Vol 5, chapter 124, Elsevier ISBN 0-7506-3372-7. 


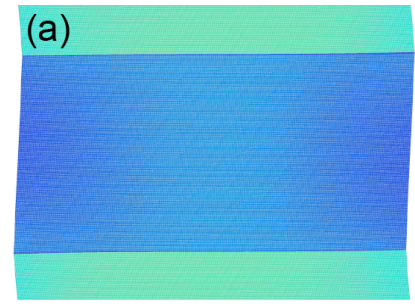

(d)
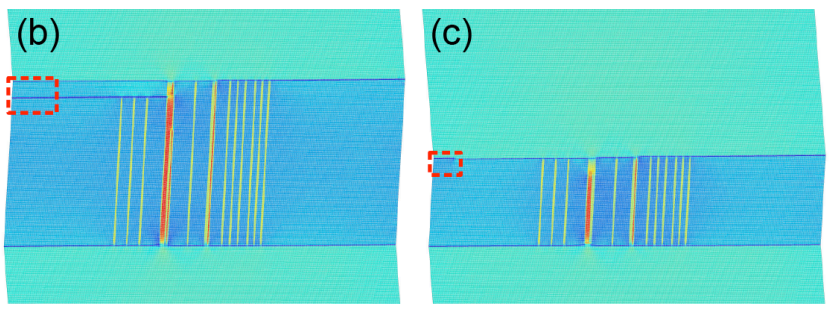

(e)

(f)

$-5.24$
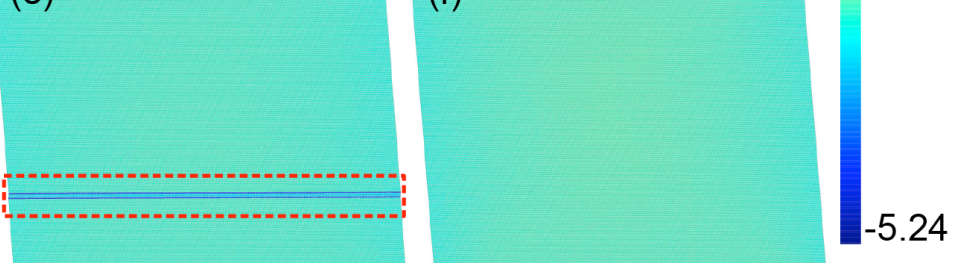

FIG. 1. Pattern evolution during shear deformation. Patterns showed in (a)-(f) correspond to the blue dots in Fig. 2 with the full time scale $t_{1}-t_{6} . t_{1}$ is the time when the upper yield point is passed. The microstructure is shown before yield in (a) and after yield in (b). At the time $t_{3}$, kinks move towards the surface (c). At times $t_{4}-t_{5}$ secondary patterns form (d, e), while at $t_{6}$ (f) the single domain state is re-established. The color scheme relates to the total shear angle between adjacent atoms. This angle is defined as $\theta=\left|\theta_{\text {ver }}\right|-4+\theta_{\text {hor. }} \quad \theta_{\text {ver }}$ and $\theta_{\text {hor }}$ denote the local shear angle in the I vertical direction and horizontal direction respectively and are calculated over three neighboring atoms.
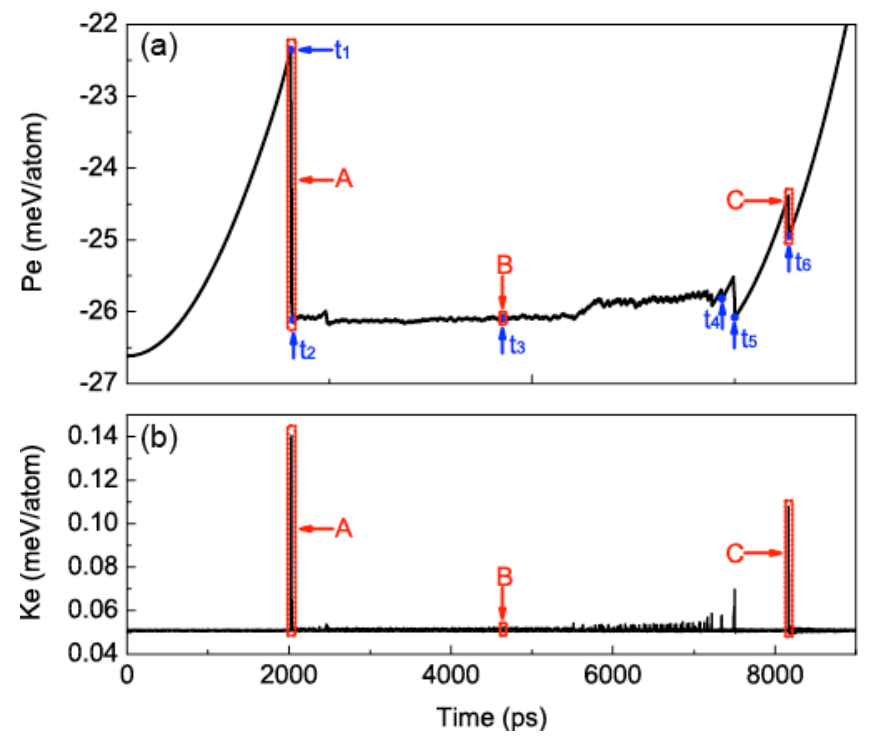

FIG. 2. Potential energy (a) and kinetic energy (b) evolution during shear deformation. The rectangle A, B, C corresponds to the yield, kink, and horizontal needle regime in Fig.1. 

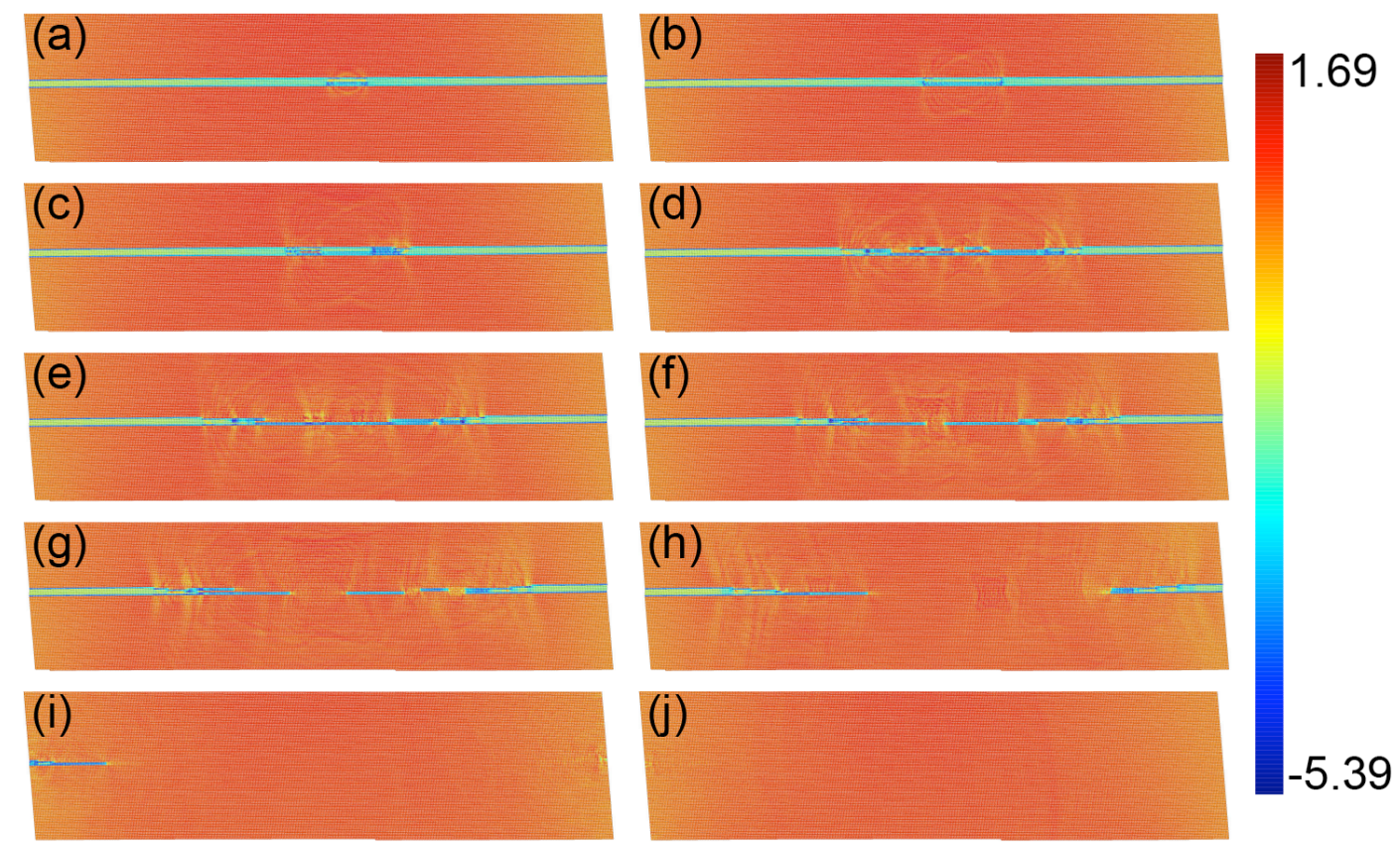

FIG. 3. Destruction of a horizontal needle domain. This spanning domain reduces first its thickness by kink movements and then breaks into smaller segments. (a) is the red rectangle region of Fig.1(e). When loadeded, these segments then shrink until they disappear and the single domain state is re-established. Note the shock waves which are emitted when vertical needles inside the horizontal needle domain move sideways. The color scheme relates to the total shear angle between adjacent atoms. This angle is defined as $\theta=\left|\theta_{\text {ver }}\right|-4+\theta_{\text {hor. }} \quad \theta_{\text {ver }}$ and $\theta_{\text {hor }}$ denote the local shear angle in the vertical direction and horizontal direction and are calculated over three neighboring atoms. 


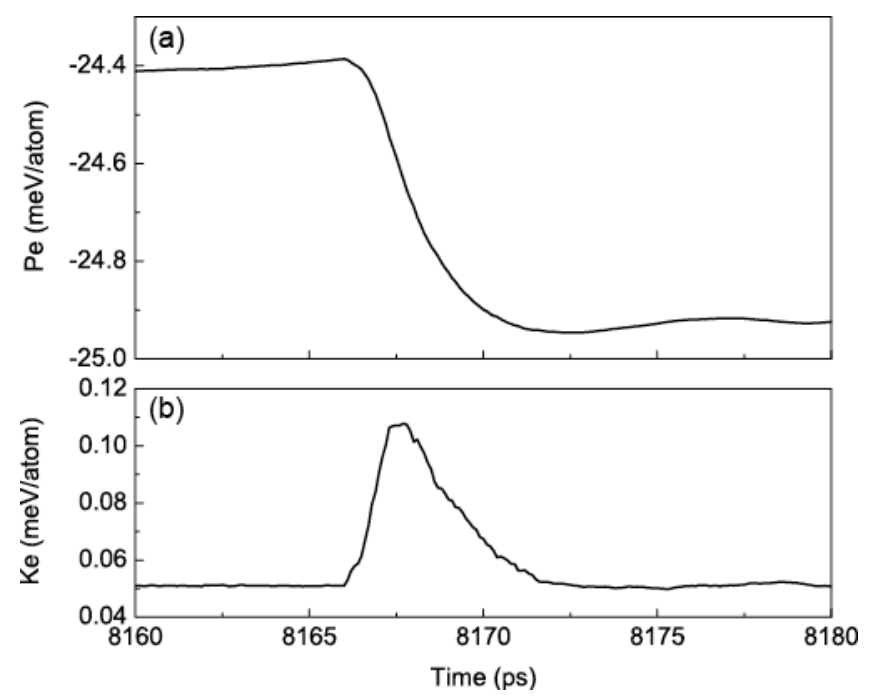

FIG. 4. Changes of the bulk potential (a) and kinetic (b) energies during the collapse of the horizontal needle domain. The potential energy is reduced by ca. $0.56 \mathrm{meV} / \mathrm{atom}$. Part of this energy leads to an increase of the kinetic energy by $0.06 \mathrm{meV} / \mathrm{atom}$ while the rest is stored in the strain energy of the sample leading to the displacements of the surface atoms. The de-pinning event with a large energy step is shown in the red box near the total de-pinning point. 

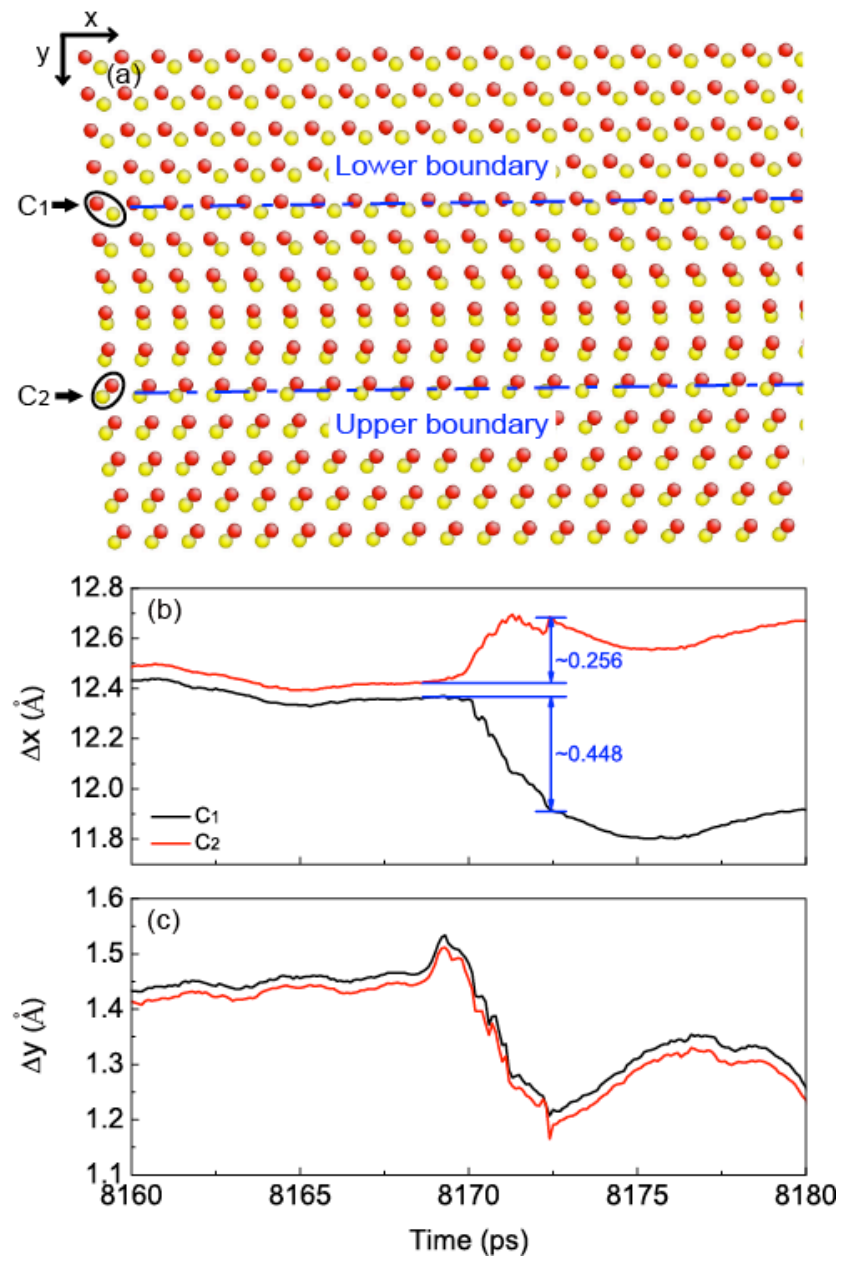

FIG. 5. (a) shift of atomic positions before(yellow) and after(red) the collapse of the horizontal needle domain. Displacements of the surface atoms $\mathrm{C}_{1}$ and $\mathrm{C}_{2}$ at the needle domain boundaries in $\mathrm{x}$ and direction (b) and $\mathrm{y}$ direction (c). 


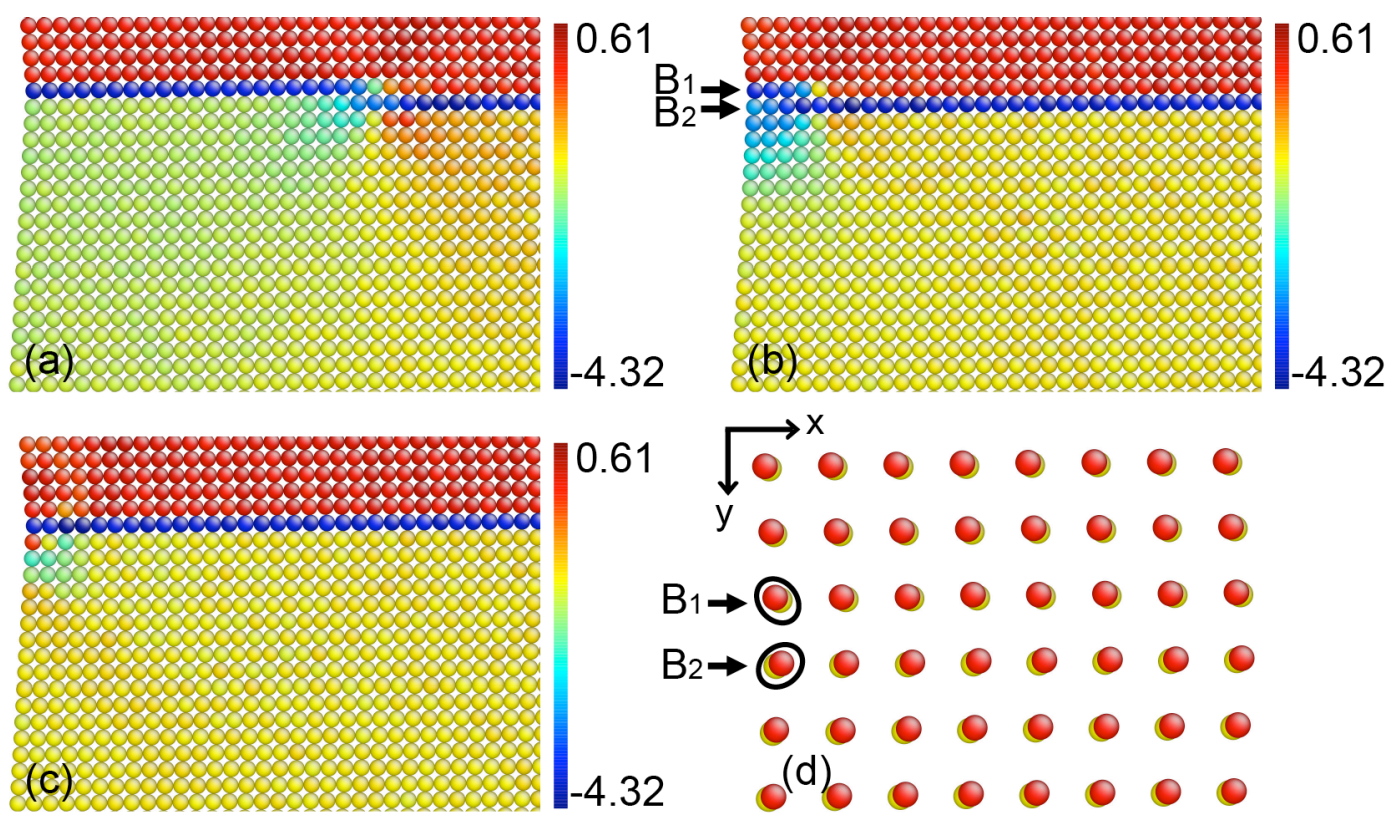

FIG. 6 Arrival of a complex kink attached to a horizontal twin domain at the surface. The kink causes a large rearrangement of the strain pattern at the surface and causes acoustic emissions.

I Fig.6(a) is the red rectangle region of Fig.1(c), showing a moving kink in the bulk of the materials, (b) the kink has moved to the surface, (c) the collision process has finished. (d) I Displacement pattern before(yellow) and after (red) the impact of one kink movement at the surface. The shift of the horizontal twin boundary requires the displacements of all atoms with the largest differential change near the intersection between the twin boundary and the surface (see Ref.50 for details). 

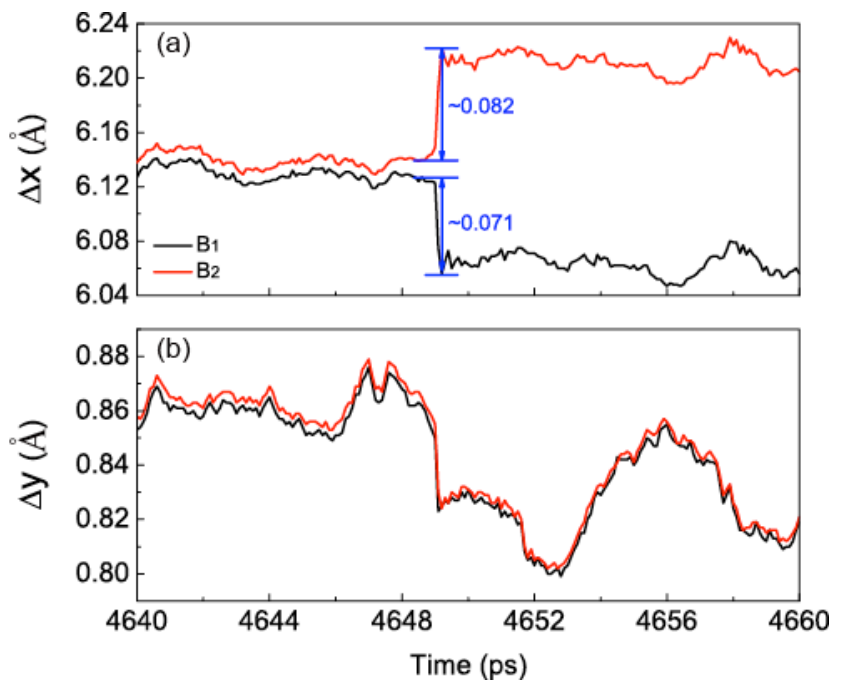

FIG. 7. Displacements of the atoms B1 and B2 in Fig.6. The total displacements relate to the full avalanche. At the beginning, atoms B1 and B2 move to the negative direction of X-axis. When the kink comes to the surface, B1 displays a displacement burst in the negative direction and B2 displays a displacement burst in the positive direction.

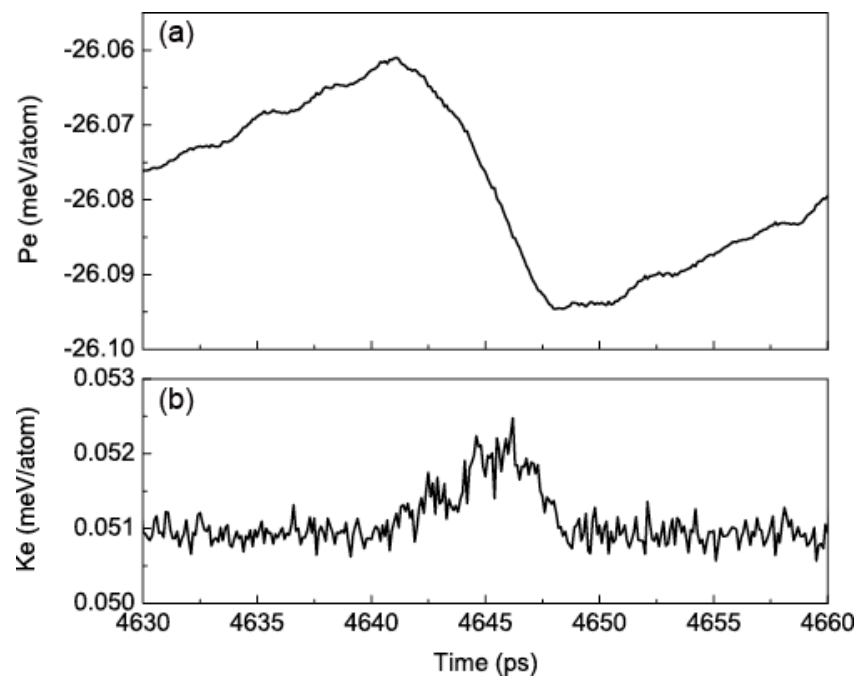

I FIG. 8. (a) Potential energy and (b) kinetic energy evolution during the collision of two kinks with the surface. The potential energy is reduced by ca.0.034meV/atom and kinetic energy increases by ca. $0.0015 \mathrm{meV} /$ atom. 

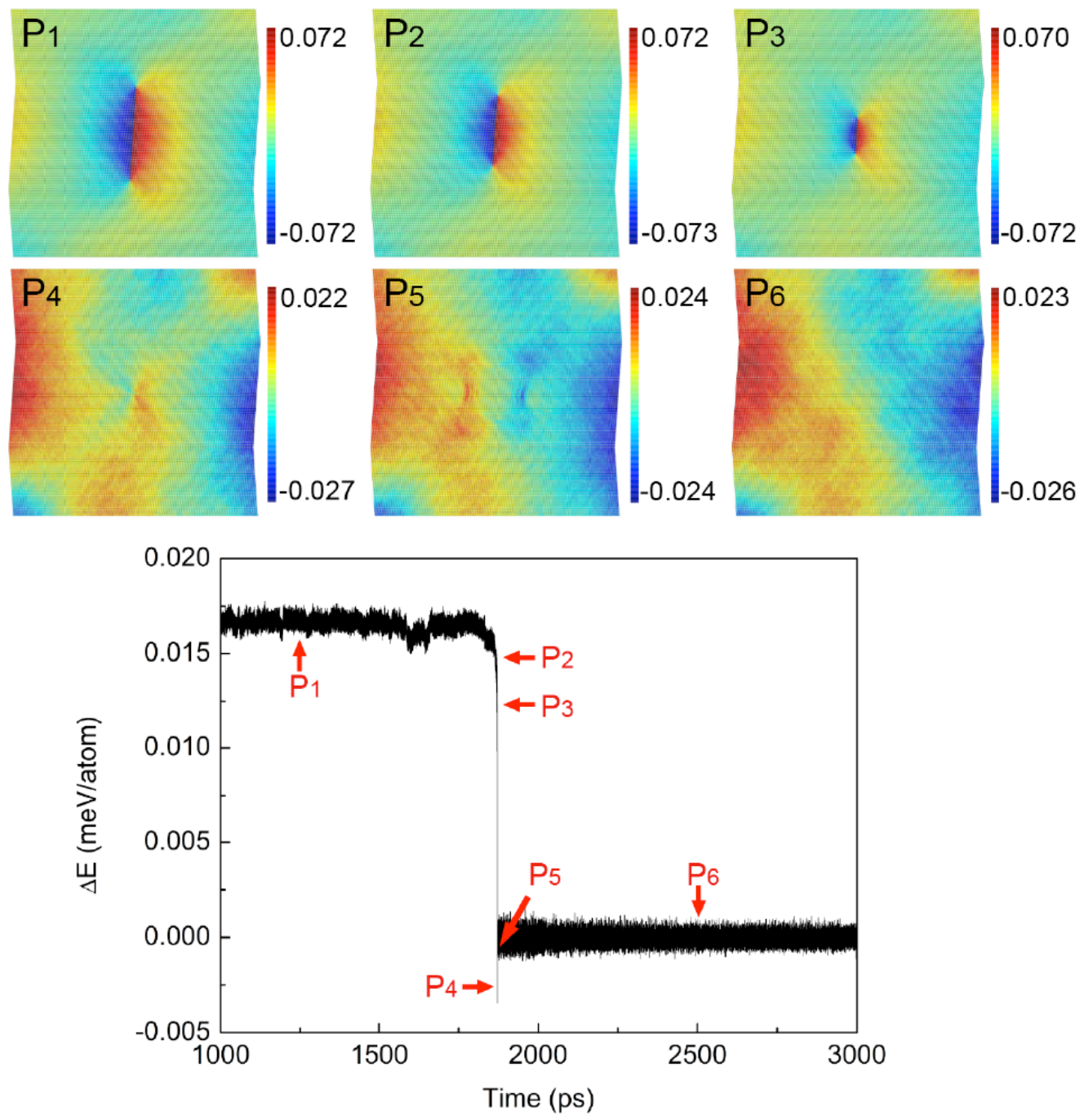

FIG. 9. Collapse of the potential energy during the annihilation of a vertical needle domain. The I upper figures P1-P6 show the shear strain patterns at various stages of the thermal anneal. Strain fields just before and after the final collapse of a vertical needle domain. Shock waves are emitted from the collapsing needle domain, the change in potential energy is shown in the lower panel. 

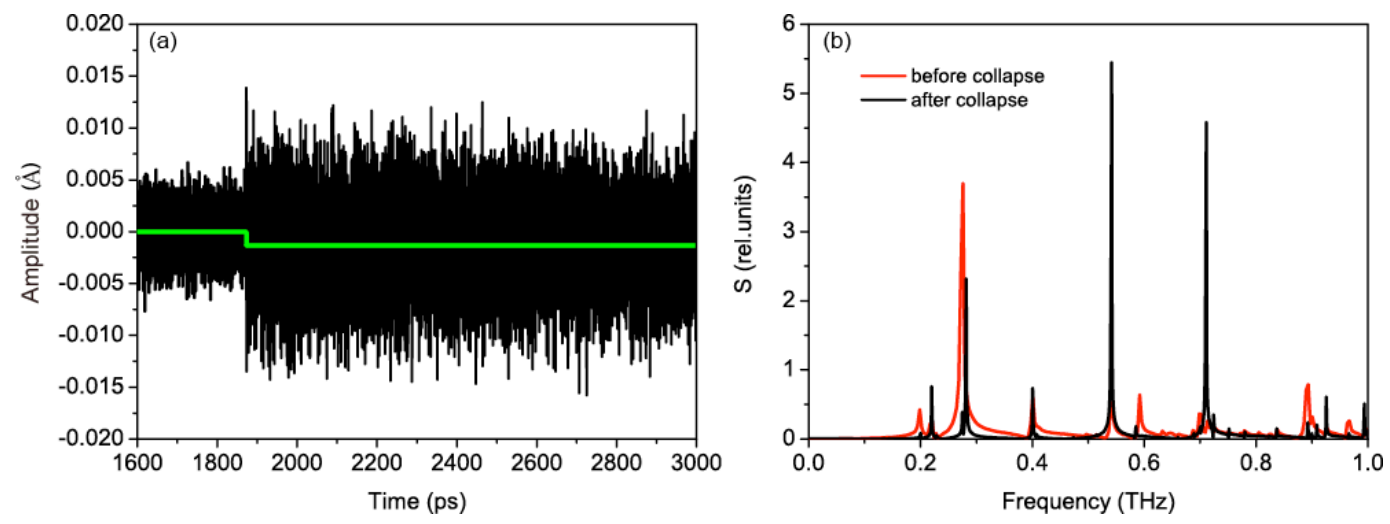

FIG. 10 (a). Vibrational amplitudes before and after the collapse point at 1870.8ps. The thermal fluctuations during the early stages before the collapse of the needle increases after the collapse and leads to the 'ringing' of the sample. The static displacement is the time-integrated means and shows a step at the collapse point, the displacement is ca. $0.0014 \AA$. (b). Vibrational spectra $S(\omega)$ before (red) and after(black) the collapse.

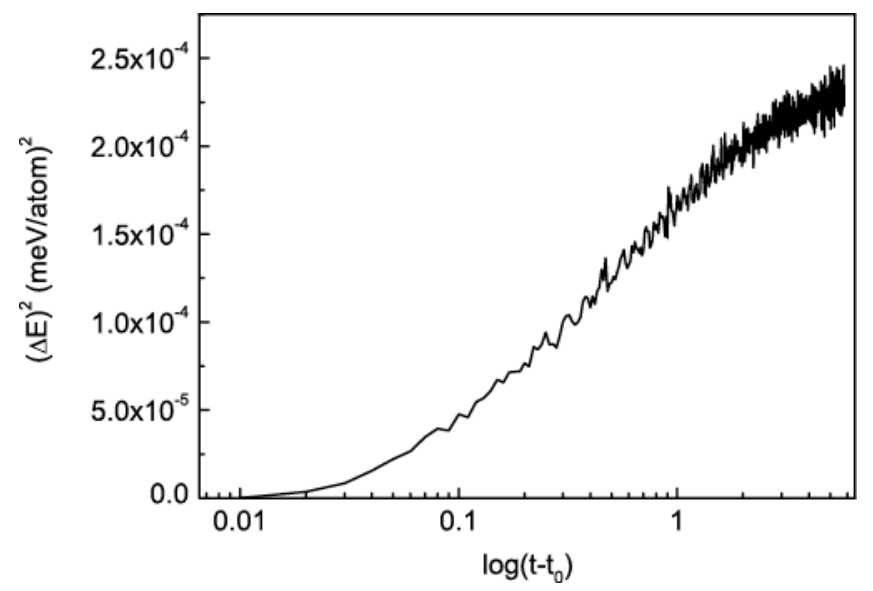

FIG. 11. Semi-log display of the squared potential energy after the irreversibility point when the I needle starts to shrink rapidly before the critical collapse at time $t_{0}$. 

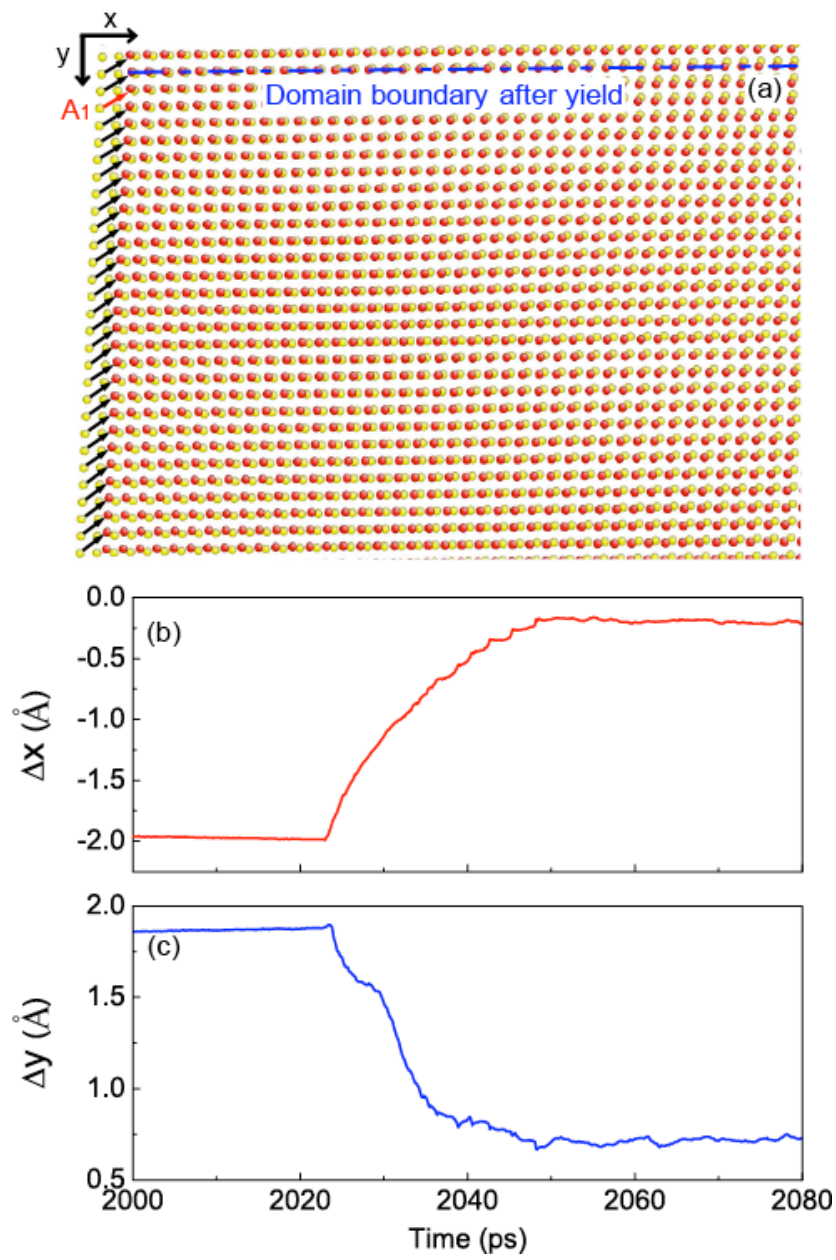

I FIG. 12. (a) shift of atomic positions before(yellow) and after(red) the yield event; Displacements of the surface atom $A_{1}$ in $x(b)$ and $y(c)$ direction 

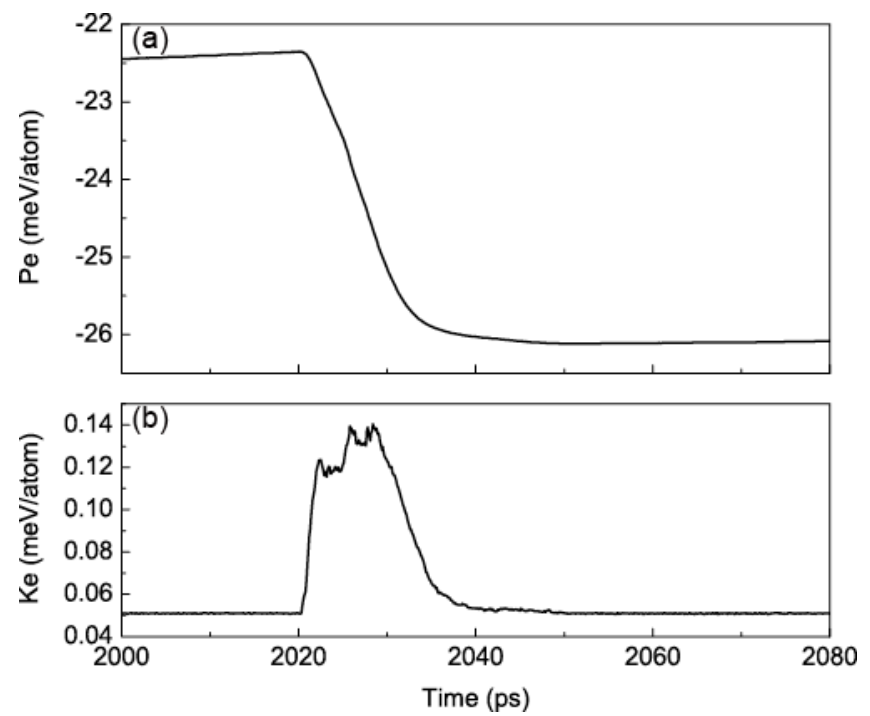

FIG. 13. Time evolution of the potential energy (a) and kinetic energy (b) during the yield event. The kinetic energy decreases by $3.7 \mathrm{meV} /$ atom and the kinetic energy increases by $0.09 \mathrm{meV} /$ atom. 